\title{
IN SITU OPHTHALMIC GEL WITH ION ACTIVATED SYSTEM
}

\author{
INSAN SUNAN KURNIAWANSYAH ${ }^{1}$, TAOFIK RUSDIANA ${ }^{1}$, HABIBAH A. WAHAB ${ }^{2}$, ANAS SUBARNAS ${ }^{3}$
}

1Department of Pharmaceutics and Pharmaceutical Technology, Faculty of Pharmacy, Universitas Padjadjaran, Sumedang, West Java, Indonesia, ${ }^{2}$ School of Pharmaceutical Sciences, Universiti Sains Malaysia, Pulau Pinang, Malaysia, ${ }^{3}$ Department of Pharmacology and Clinical Pharmacy, Faculty of Pharmacy, Universitas Padjadjaran, Sumedang, West Java, Indonesia

Email: insan.sunan.kurniawansyah@unpad.ac.id

Received: 14 Mar 2019, Revised and Accepted: 24 May 2019

\section{ABSTRACT}

In situ gel with ion activated system which occurs as triggered by a change in the ionic strength. Osmotic gradient is a factor that determines the rate of gelation on the surface of the gel. Polymers play an important role in drug delivery from their dosage forms. Polymeric in gelling systems provides longer drug release compared to conventional delivery systems. The use of biodegradable and biocompatible polymers for in situ gel formulation makes the drug delivery system acceptable and controlled. Thus the continuous and prolonged release of the drug, biocompatibility characteristics makes the dose gel form in situ reliable. Polymers that are used in ion activated in situ gelation can be various, such as gelrite gellan gum, alginates, deacetylated gellan gum, anionic polymers (carbopol), cationic polymer (chitosan), non-ionic polymers (HPMC, Methylcellulose), thiolated polymer (thiomers), carbomer (polymer used in ophthalmic), polycarbophil (polymer used in ophthalmic). This review is written based on the data or information obtained by using several search engines and several scientific journals, using the keywords in situ gel with polymers, ion activated the system, and limited search years in 2010 and above.

Keywords: In situ gel, Ion activated system, Biodegradable and biocompatible polymers

(C) 2019 The Authors. Published by Innovare Academic Sciences Pvt Ltd. This is an open access article under the CC BY license (http://creativecommons.org/licenses/by/4.0/) DOI: http://dx.doi.org/10.22159/ijap.2019v11i4.33072

\section{INTRODUCTION}

The goal of pharmacotherapeutics is to treat a disease in a consistent and predictable fashion. In topical application, the drops provide therapeutically effective concentrations that have been the preferred route of administration for bacterial conjunctivitis and keratitis because the drops wash away bacteria and bacterial antigens; adverse systemic effects of the drugs are decreased or eliminated [1]. The drug development with new dosage forms has always been done to provide effective and easy to use by patients. In addition, the presence of new drug preparations may increase bioavailability and reduce side effects. One of the discovery is a breakthrough of gel preparations with unique characteristics, such as in situ gel. Over the last decades, an impressive number of novel temperature, $\mathrm{pH}$, and ion-induced in situ forming solutions have been described in the literature. Each system has its own advantages and drawbacks. The choice of particular hydrogels depends on its intrinsic properties and investigated therapeutic use [2].

The ocular residence time of the drug needs to increase for the ocular bioavailability of drug that increases. In situ forming gels are formulations, where they undergo a transition into a gel, conveniently dropped in the eye, applied as a solutions [3, 4], or suspensions. In situ gelling systems developing are to prolong the precorneal residence time of a drug, consequently, enhance ocular bioavailability, and improve patient compliance. The in situ gel are more acceptable for the patients and has been the most attractive feature systems caused of the precorneal residence time of some in situ gelling for several hours [5].

The cornea as the main barrier for the ocular absorption of drugs, where is that acts as a barrier for penetration of hydrophilic drug is the outer epithelium layer while that acts as a barrier for the hydrophobic drug is the inner stroma. Develop an ophthalmic novel drug system without damaging the natural mechanism and barriers of the eyes at the same time achieving affective ocular drug concentration at the site of action while improving the residence time of drugs in eyes is the solution for the small portion drug will be lost on the anterior segment of the eyes [6].

The advantages of in-situ forming polymeric delivery systems are ease and reduced frequency of application, comfort, and improved patient compliance [7]. In situ gel formation occurs due to one or combination of different stimuli like $\mathrm{pH}$ change, temperature modulation using polymers, solvent exchange employing polymers [7], fungal keratitis that must be diagnosed and treated affectively, previous ocular surface disease and trauma as the leading causes of fungal infection in the cornea, prolonged chemo-or immune-suppressive therapy, contact lens use, current therapeutic options are limited, and oral therapy requires high doses of antifungal agents which may cause unwanted side effects so by use of in situ gels can resolve the problem of short residence time of formulations on the eye surface [8].

\section{In situ gel with ion activated system}

\section{Ion activated systems}

Polymers may undergo a phase transition in the presence of various ions. Some of the polysaccharides fall into the class of ion-sensitive polymers. Alginate is used as the gelling agent in combination with Hydroxy Propyl Methyl Cellulose/HPMC (Methocel E50Lv) which acted as a viscosity enhancing agent. Gelrite gellan gum, a novel ophthalmic vehicle that gels in the presence of mono or divalent cations, present in the lachrymal fluid can be used alone and in combinations with sodium alginate as the gelling agent. Gelrite is an anionic polysaccharide that undergoes in situ gelling in the presence of mono and divalent cations, especially $\mathrm{Ca}^{2+}$. Likewise, alginic acid undergoes gelation in presence of divalent/polyvalent cations due to the interaction with guluronic acid block in alginate chains [9].

Table 1: Various in situ gel mechanisms along with the polymer

\begin{tabular}{lll}
\hline External stimuli & Mechanism & Examples of polymer \\
\hline $\begin{array}{l}\text { Temperature } \\
\text { dependent system } \\
\text { pH-triggered system }\end{array}$ & $\begin{array}{l}\text { Formulation is liquid at room temperature }\left(20-25{ }^{\circ} \mathrm{C}\right) \text { which } \\
\text { undergoes gelation in contact with body fluid }\left(35-37^{\circ} \mathrm{C}\right)\end{array}$ & $\begin{array}{l}\text { Poloxamer/Pluronics, Cellulose } \\
\text { derivative }\end{array}$ \\
$\begin{array}{ll}\text { Phase transition occur due to rise in pH from } 4.2 \text { to } 7.4 \\
\text { Ion activated system }\end{array}$ & $\begin{array}{l}\text { Pseudolatexes, Carbomer (Acrylic acid) } \\
\text { Cellulose acetate phthalate latex } \\
\text { influence of an increase in ionic strength }\end{array}$ & $\begin{array}{l}\text { Chitosan, Gellan gum/Gelrite, Alginate } \\
\text { 1,2,3,7,9 }\end{array}$ \\
\hline
\end{tabular}

From table above, we know that polymers used in ion activated system were chitosan, gallen gum, and alginate. Which mechanism is undergoing liquid-gel transition under the influence of an increase in ionic strength [1]. 


\section{Reported ophthalmic in situ gel with ion activated system}

Polymers that are used in ion activated in situ gelation can be various, such as gelleriete gellan gum, alginates, deacetylated gellan gum, anionic polymers (carbopol), cationic polymer (chitosan), nonionic polymers (HPMC, Methylcellulose), thiolated polymer (thiomers), carbomer (polymer used in ophthalmic), polycarbophyl (polymer used in ophthalmic) and many more.

Placebo in situ gelling systems were prepared using different polymer ratios and evaluated for their gelling capacity to identify a suitable composition. Chitosan was dissolved in acetate buffer, $\mathrm{pH}$ 5.5-6.0. Gellan gum was dissolved in ultrapure water with heating at $70-80{ }^{\circ} \mathrm{C}$ with stirring. The gelling capacity was determined by placing a drop of the system in a vial containing $2 \mathrm{ml}$ of freshly prepared simulated tear fluid (STF) using sodium chloride $0.670 \mathrm{~g}$, sodium bicarbonate $0.200 \mathrm{~g}$, calcium chloride dihydrate $0.008 \mathrm{~g}$ and purified water q. s. $100 \mathrm{~g}$ and visually assessing the gel formation, noting the time taken for gelation and time taken by the formed gel to dissolve [10].

The formulation and evaluation of in situ ocular gelling systems (ion activated gelling systems) of Ketorolac tromethamine have done. These gelling systems involve the use of Gelrite as polymer. They evaluated the formulation for clarity, $\mathrm{pH}$ measurement, gelling capacity, drug content estimation, rheological study, in vitro drug release, ocular irritancy studies (as per draize test) and ex-vivo corneal permeation studies using isolated goats cornea. The developed formulations showed the sustained release of drug for up to $6 \mathrm{~h}$. The formulations were found to be non-irritating with no ocular damage [11].

The preparation and characterization of in situ gel were performed using chitosan (water soluble) and sodium glycerophosphate was dissolved in distilled water separately at room temperature. Complete solvation was achieved by placing the solutions in the refrigerator for $2 \mathrm{~h}$. The glycerophosphate solution was then added dropwise into the chitosan solution with constant stirring. Pilocarpine hydrochloride $(2 \% \mathrm{w} / \mathrm{v})$ was dissolved in small volume of distilled water and then added to the polymeric solution with stirring. The stirring was continued for $10 \mathrm{~min}$ to gain homogenous mixture. Benzalkonium chloride was added as a preservative in the preparation. The sterilization was carried out by exposing in situ gels filled in glass vials to ultraviolet (UV) radiations in a UV chamber for a period of $60 \mathrm{~min}$ at room temperature $\left(25^{\circ} \mathrm{C}\right)$ in air. The characterization that done were gelation temperature, gelation time, isotonicity test, determination of viscosity, determination of drug content, in vivo and in vitro studies [12].

In situ gel from Sesbania grandiflora flower extract for the treatment of bacterial conjunctivitis was prepared and evaluated too. For the preparation of gel dispersed the required amount of Pluronic F127 and chitosan was dispersed in cold distilled water and $0.2 \%$ acetic acid stirred it for 1 hour. Pluronic solution was placed in the refrigerator until it dissolves completely. The extract was weighed and dissolved it in a small amount of water. Chitosan solution and solution of extract was added and stirred it vigorously for 1 hour. After that, the remaining ingredients were added while stirring. They prepared the gel containing a various concentration of extract and the formulation codes are from G1 to G6. Evaluation that done were physical evaluation, measurement of $\mathrm{pH}$, gelling capacity, gelation temperature and rheological study [13].

Cao et al. has prepared ion-activated in situ gel systems of scopolamine hydrobromide and evaluated its anti-motion sickness efficacy. Preparation of nasal formulations A certain amount of gellan gum was added to deionized water and dissolved by heating to $100{ }^{\circ} \mathrm{C}$ with moderate stirring. After cooling to below $40{ }^{\circ} \mathrm{C}$, Scopolamine (SCOP) $(0.4 \%, \mathrm{w} / \mathrm{v})$, mannitol $(5 \%, \mathrm{w} / \mathrm{v})$, and chlorhexidine acetate $(0.01 \%, \mathrm{w} / \mathrm{v})$ were added and mixed well. Three kinds of SCOP in situ gels were prepared at the concentrations of gellan gum which were $0.2 \%, 0.5 \%$, and $1.0 \%$ (w/v), respectively. The $\mathrm{pH}$ of all the formulations was between 4.0 and 6.0 [14].

Balasubramaniam and Pandit prepared and evaluated ion-activated in situ gelling systems for sustained ophthalmic delivery of ciprofloxacin hydrochloride $(\mathrm{CPH})$. The preparation was: gellan alone and its combinations with sodium alginate, with or without sodium citrate $(20 \% \mathrm{w} / \mathrm{w})$, were dissolved in hot acetate buffer $(70$ ${ }^{\circ} \mathrm{C}$, prepared in fresh water for injection under laminar flow) $\mathrm{pH} 5.0$ by continuous stirring at $40^{\circ} \mathrm{C}$. The required quantity of $\mathrm{CPH}$ to give a final drug concentration of $0.3 \% \mathrm{w} / \mathrm{v}$ was added to the polymeric solution and stirred until dissolved. The formulations were filled in $10 \mathrm{ml}$ amber colored glass vials, capped with rubber closures, and sealed with aluminum caps. The formulations, in their final pack, were terminally sterilized by autoclaving at $121^{\circ} \mathrm{C}$ and 15 psi for 20 min. The sterilized formulations were stored in a refrigerator (4-8 ${ }^{\circ} \mathrm{C}$ ) until further use. The evaluation that done were drug content uniformity, gelation studies, rheological studies, in vitro release studies, antimicrobial efficacy studies, in vivo rabbit eye study [15].

Swapnil et al. designed and evaluated Ion Induced in situ gel formulation for levofloxacin hemihydrate ocular delivery. This type of formulation was prepared in deionized water as the gelling agent forms a stiff gel when interact with the ions in the buffer system. Take $0.9 \% \mathrm{w} / \mathrm{v}$ of sodium chloride and add this under constant stirring, then add benzalkonium chloride $(0.02 \% \mathrm{w} / \mathrm{v})$ to above the solution as a preservative. After that add levofloxacin solution $(0.5 \%$ $\mathrm{w} / \mathrm{v}$ ) in polymer solution under stirring to form uniform solution and lastly make up the volume up to $100 \mathrm{ml}$. Formulations were tested for ocular irritation study [16].

Sneha et al. prepared and evaluated ion activated in situ gel of gellan gum containing salbutamol sulphate for nasal administration. The preparation was accurately weighed the quantity of gellan gum $0.2-$ $0.5 \% \mathrm{w} / \mathrm{v}$ ) was dispersed in distilled water. The dispersions were then stirred for $20 \mathrm{~min}$ at $85-90{ }^{\circ} \mathrm{C}$ using magnetic stirrer and then cooled to room temperature. HPMC E15 was added slowly with stirring. d-mannitol, benzalkonium chloride and Tween 80 were also added simultaneously. Finally, salbutamol sulphate was added with stirring. The $\mathrm{pH}$ of all formulations was in the range of 4.5-6.5. Formulations were filled in amber colored glass vials, capped with rubber closures and sealed with aluminium caps. Formulations were stored in a refrigerator $\left(4-8{ }^{\circ} \mathrm{C}\right)$ until further use. Evaluation that done were in vitro gelation study, viscosity measurements, drug content determination, gel strength determination, in vitro mucoadhesive strength, in vitro drug release study, ex vivo permeation study, histopathological studies, stability study [17].

Polymers play an important role in drug delivery from their dosage forms. Polymeric in gelling systems provides longer drug release compared to conventional delivery systems. A variety of synthetic, synthetic polymers are developed for controlled drug release. The use of biodegradable and biocompatible polymers for in situ gel formulations makes the drug delivery system acceptable and controlled. Thus the continuous and prolonged release of the drug, the biocompatibility characteristics make the dose gel form in situ reliable. In the latest technology, the polymer combination focuses on the development of safe ophthalmic delivery systems [18].

Polymers that are used in ion activated in situ gelations can be various, such as gelleriete gellan gum, alginates [19], deacetylated gellan gum, anionic polymers (carbopol), cationic polymer (chitosan), non-ionic polymers (HPMC, Methylcellulose), thiolated polymer (thiomers) [20], carbomer (polymer used in ophthalmic), polycarbophil (polymer used in ophthalmic) [21,22], and many more.

Gellan gum is anionic heteropolysaccharide, which forms a clear gel in the presence of mono ordicalent cations. The electrolytes of the tear fluid and especially $\mathrm{Na}^{+}, \mathrm{Ca} 2+$ and $\mathrm{Mg} 2+$ cations are particularly suited to initiate gelation of the polymer when instilled as a liquid solution into the cul-de-sac. There is cross-linking of negatively charged polysaccharide and cations. Once gelled, the formulation resists the natural drainage process from the precorneal area [20].

Sodium alginate is a natural hydrophilic sodium salt of alginic acid and forms three-dimensional hydrogel matrics with low viscosity, free-flowing liquid at concentrations suitable for gel formation in the lachrymal fluid. Alginate changes into the stable gel phase due to ionic crosslinking of alginate chains with tear ca $2+$. To decrease the amount of alginate required for gelation, HPMC was mainly used as viscosity enhancer [22]. 
Every polymers are made in situ hydrogel dosage form as its base in some different formulation. These polymers are tested and determine the best polymers formulation for this dosage form through some evaluation, such as physical parameters, gelling capacity, rheological studies, in vitro drug release studies, texture analysis, isotonicity evaluation, ocular irritancy test, accelerated stability studies, sterility studies, sol-gel transition temperature and gelling time [19,23], gelling ability, drug content, swelling studies, FTIR [21, 22], permeation studies across a sheep's corneal membrane [21].

In the case of gellan containing preparations, increasing the concentration of gellan beyond $0.0625 \%$ caused gelation upon cooling to $40{ }^{\circ} \mathrm{C}$ (during stirring). The ionic content of the vehicle used, in this case, acetate buffer $\mathrm{pH} 5.0$, and the presence of $\mathrm{HCl}$ in $\mathrm{CPH}$ could have contributed to the gelation of gellan when used beyond $0.0625 \%$ (used in this study). In the combination systems with sodium alginate, the concentration of gellan was kept constant at $0.03 \%$ and the concentration of sodium alginate was varied to give a maximum of $1 \%$ polymer concentration since an increase in total polymer concentration beyond $1 \%$ resulted in gelation during formulation [8]. The rheological studies result showed that gellan alone didn't make any change in viscosity after autoclaving, in the other hand, the formulation contains a combination of sodium alginate and gellan showed around $10-15 \%$ reduction in viscosity after autoclaving. The in vitro release study of all concentration showed that the amount of drug released in the first minute decreased with increasing polymer concentration and this trend continued for the entire duration of the study. The last test is in vivo rabbit eye studies. The tested formulations showed a markedly improved effect when compared with the marketed (standard) eye drop. The formulations were successful in inhibiting the growth for the entire duration of the study in all the animals infected with $P$. aeruginosa. The formulations used for the in vivo study formed a translucent gel immediately after installation into the eye. Gross examination of the ocular tissues showed that the formulations did not cause undue irritation and no leakage of the gelled material was seen from any part of the eye [15].

The study of using a combination of $0.5 \%$ gellan gum and $0.25 \%$ chitosan as the polymer for in situ gel base was done. Chitosan is reported to act as penetration enhancers that increase transcorneal permeation of the drug. Besides this, its other properties like bioadhesiveness, viscous nature, and ability to convert into a hydrogel at $\mathrm{pH}>6.5$ make it the best suitable candidate for the development of this type of delivery systems [24]. Interaction studies were carried out to check any possible interaction between formulation ingredients. UV spectra obtained before and after autoclaving were found to be identical. No additional peak emerged or shift in the existing peak occurred. It revealed that the ingredients were compatible to each other and no physicochemical reactions took place. Ocular irritation of the developed formulation was checked by Hen's egg chorioallantoic membrane test which is a rapid, sensitive, and inexpensive test. Testing with incubated eggs is a borderline case between in vivo and in vitro systems and does not conflict with the ethical and legal obligations. This study showed that the formulation was non-irritant to mild irritant and it's well tolerable [10].

Many studies about in situ gel with ion activated systems. Research about development and characterization of in situ gel for an ophthalmic formulation containing ciprofloxacin hydrochloride showed in the FTIR that no interaction between polymer and ciprofloxacin $\mathrm{HCl}$ drugs. The $\mathrm{pH}$ of the gel solution in situ was found to be about 6.49-6.58 for all formulations. The IG 3 formulation (sodium alginate: $\mathrm{HPMC}=1.5: 0.5$ ) has $6.53 \mathrm{pH}$ which is an acceptable range for eye preparations. Rheology research showed that HPMC addition showed good results ciprofloxacin $\mathrm{HCl}$ was successfully formulated as in situ gel-forming eye drops using Na-alginate and HPMC which can increase bioavailability and patient compliance. Physicochemical characterization research and drug release in vitro indicate that the development of developed formulations can be an alternative to conventional eye drops and ointments in ease and with the added benefit of sustain released resulting in improved patient compliance [25].
In the research of formulation and evaluation in situ mucoadhesive ophtalmic hydrogels for sustained delivery of pefloxacin mesylate, optimation of in situ gel with ion activated the system from pefloxacin mesylate can be formulated using a combination of sodium alginate and hydroxyethylcellulose. Liquid formulation at non physiological $\mathrm{pH}[\mathrm{pH}$ 5] and experiencing rapid gelation at physiological $\mathrm{pH}$ [pH 7.4]. That experiment showed it has good bioadhesive, viscosity and kinetic release research showed sustained release in 1 period of $12 \mathrm{~h}$ followed by reaction of order 0 . Isotonic optimized formulation, not irritate, and showed antimicrobial efficacy. It can be concluded that the formulations obtained show that the preparation of the formulation is easily managed in the form of drops undergoing a transition phase to form a viscellable gel whose residence time is precorneally increased and the drug lasts for a longer time [26].

Salunke and Patil said that deacetylated gellan gum was selected in comparison with high acetylated form because it has desirable properties such as responsible for ion activated gelation, having low viscosity, clear in clarity, firm and brittle structure. All gellan gum formulations showed immediate gelation within a period of 10-15 seconds, depending upon the concentration of gellan gum used. The formulation containing $0.3 \%$ and $0.4 \%$ of gellan gum showed optimum gelation but the formulation containing $0.3 \%$ of gellan gum showed satisfactory viscosity for spraying as a droplet, so the $0.3 \%$ of gellan gum in the formulation showed optimum viscosity in the sol-gel transition state. This formulation was also safe to the surface of epithelium lineng and the granular cellular structure of the nasal mucosa [17].

Another polymer that can be used as gel-based is gelrite. Gelrite is made in 3 concentration $(0.5 \%, 0.75 \%$, and $1 \%)$ using ketorolac tromethamine as active compound. The result shows that in the concentration of $0.75 \% \mathrm{w} / \mathrm{v}$, gelrite was found to be a better carrier system since it shows optimum gelation. The increase of gelrite concentration makes the gelation capacity also increases. In the in vitro dissolution test, simulated tear fluid (basic media at $\mathrm{pH}$ 7.4) was selected as media for the dissolution studies. Ion activated ocular gel with $0.75 \% \mathrm{w} / \mathrm{v}$ of Gelrite concentrations showed the highest dissolution rate $(86.96 \%$ within $6 \mathrm{~h})$. The ocular irritancy studies indicate that the formulation was a non-irritant and very well tolerated by the eye. No ocular damage or abnormal clinical signs to the cornea, iris, or conjunctivae were visible. Corneal permeation studies were performed using isolated goat's cornea on Franz diffusion cell using STF ( $\mathrm{pH} 7.4$ ) at $34 \pm 0.5^{\circ} \mathrm{C}$. the result is the percentage of drug release within $5 \mathrm{~h}$ is $86.79 \%$ [27].

In the other experiment, gelrite polymer also tested in various concentration between $0.2 \%-0.8 \%$. Clarity of all formulations was found to be satisfactory. The $\mathrm{pH}$ within an acceptable range and hence would not cause any irritation upon administration of the formulation. The result of drug content for all formulation was found to be in acceptable range $(72-86 \%)$. The formulation F6 $(0.7 \%)$ has an optimum viscosity (1985 cps) before gelation which will allow its installation into the eye as a liquid this will then undergo rapid solgel transition due to ion exchange and the viscosity after gelation was found to be $4520 \mathrm{cps}$. The results of F6 in vitro release studies showed better sustaining effect amongst all formulation. This may be due to the higher concentration of gelrite. The formulation F6 passed the sterility test (no turbidity in more than $7 \mathrm{~d}$ ) [28].

Sodium alginate and carbopol also can be used as gel based. In situ gel forming systems were prepared using sodium alginate and carbopol 934 at different concentrations along with HPMC as a viscosity enhancing agent. The maximum concentration of sodium alginate used was $2 \%$ and carbopol was $0.12 \%$ to develop gel systems of flurbiprofen $(0.03 \%$ in all formulation). The result can be concluded that polymer combination $2 \%$ alginate and $0.4 \%$ HPMC showed better potential for sustained topical drug delivery to eyes for rational drug therapy as supported by in vivo test studies [22]

To achieve effective ocular therapy, a sufficient number of active ingredients must be delivered and maintained at the site of action in the eye. To increase the availability of eye medication, there is a great effort directed to the newer drug delivery system for eye administration. Conventional delivery systems often produce poor bioavailability and 
therapeutic responses because of high fluid replacement of tears and dynamics leads to rapid removal of the drug from the eye. New research in the drug delivery system is directed at incorporating several drug delivery technologies, which include building systems that not only prolong delivery contact time on the ocular surface but which at the same time slows down the elimination of the drug. There are various new dosage forms such as Insitu gel, collagen shield, minidisc, ocular film, ocusert, nanosuspension, nanoparticulate system, liposome, niosome, dendrimer, octopus, etc. Thus, to overcome the bioavailability problem, in situ intalmic gel was developed. The various temperatures, $\mathrm{pH}$, and ions that induce in situ polymeric systems used to achieve long contact time with the cornea and increase its bioavailability make optimizing ocular therapy [29].

Although various formulations exist in the market for ocular drug delivery but still unable to provide the highest bioavailability associated with the given dose. Whenever ophthalmic drugs are applied through conventional dosage forms to the anterior segment of the eye, only a small number (5\%) actually penetrate the cornea and reach the interior tissue of the eye. Affecting factors the bioavailability of the drug include rapid solution drainage by gravity, induced lachrymation, flashing reflexes, normal tear shift, superficial drug absorption to the conjunctiva and sclera, rapid removal by peripheral blood flow and low corneal permeability (acting as a barrier lipids). Advances have been made in gel technology for the development of droppable gels. They are liquid as they gradually and undergo phase transitions in the ocular cul-de-sac to form viscoelastic gels and this responds to environmental changes [30]. The systemic absorption of the drug dried through the nasolacrimal ducts may cause some undesirable side effects. To overcome these problems, inhthalmic eye gel has been investigated in an attempt to extend the ocular dose of drugs for topical applications in the eye [31].

\section{CONCLUSION}

An osmotic gradient is a factor that determines the rate of gelation and polymers play an important role in drug delivery from their dosage forms. Polymeric in gelling systems provides longer drug release compared to conventional delivery systems.

\section{AUTHORS CONTRIBUTIONS}

All the author have contributed equally

\section{CONFLICT OF INTERESTS}

\section{Declared none}

\section{REFERENCES}

1. Patil AP, Tagalpallewar AA, Rasve GM, Bendre AV, Khapekar PG. A novel ophthalmic drug delivery system: in-situ gel. Int J Pharm Res Sci 2012;3:2938-46.

2. Kurniawansyah IS, Sopyan I, Wathoni N, Fillah DL, Praditya RU. Application and characterization of in situ gel. Int J Appl Pharm 2018;10:34-7.

3. Rathor KS. In situ gelling opthalmic drug delivery system: an overview. Int J Pharm Pharm Sci 2010;2:30-4.

4. Kashikar VS, Gonjari ID. In situ gelling systems of ofloxacin: Comparative performance of in vivo precorneal drainage and pharmacokinetic study. Asian J Pharm 2013;7:15-20.

5. Patil PR, Shaikh SS, Shivsharan KJ, Shahi SR. In situ gel: a novel drug delivery system. Indo Am J Pharm Res 2014;4:5406-14.

6. Balasingam R, Khan A, Thinakaran R. Formulation of in situ gelling system for ophthalmic delivery of erythromycin. Int J Stud Res Tech Manag 2017;5:1-8.

7. Kurniawansyah IS, Sopyan I, Aditya WA, Nuraini H, Alminda FD, Nurlatifah A. Performed gel vs in situ gel: a review. Int Res J Pharm 2018;9:1-5.

8. Khatera NAA, Soliman OA, Mohamed EA. In-situ gelling ophtahlmic formulations for sustained release and enhanced ocular delivery of fluconazole. IOSR J Pharm Biol Sci 2016;11:43-51.

9. Sonawane SD, Patil RY, Lad M. A review on polymers used in novel in situ gel formulation for ocular drug delivery and their evaluation. J Biol Sci Opinion 2013;1:132-7.
10. Gupta H, Velpandian T, Jain S. Ion-and pH-activated novel insitu gel system for sustained ocular drug delivery. J Drug Targeting 2010;8:499-505.

11. Sirish V, Sadhna K, Nalini S. Formulation and evaluation of ion activated ocular gels of ketorolac tromethamine. Int J Curr Pharm Res 2010;2:54-62.

12. Venkatesh MP, Purohit KL, Kumar TMR. Development and evaluation of chitosan-based thermosensitive in situ gels of pilocarpine. Int J Pharm Pharm Sci 2013;5:164-9.

13. Vijay D Wagh, Ketaki H Deshmukh, Kalpana V Wagh. Formulation and evaluation of in situ gel drug delivery system of Sesbania grandiflora flower extract for the treatment of bacterial conjunctivitis. Int J Pharm Sci Res 2012;4:1880-4.

14. Cao S, Zhang Q, Jiang X. Preparation of ion-activated in situ gel systems of scopolamine hydrobromide and evaluation of its anti-motion sickness efficacy. Acta Pharmacol Sin 2007;28:584-90.

15. Balasubramaniam J, Pandit JK. Ion-activated in situ gelling systems for sustained ophthalmic delivery of ciprofloxacin hydrochloride. Drug Delivery 2003;10:185-91.

16. Swapnil D, Sonawane, Lahoti S. Design and evaluation of ioninduced in situ gel formulation for levofloxacin hemihydrateocular delivery. Int J Pharm Sci Invention 2014;3:38-43.

17. Salunke SR, Patil SB. Ion activated in situ gel of gellan gum containing salbutamol sulphate for nasal administration. Int J Bio Macromol 2016;87:41-7.

18. Tinu TS, Litha Thomas, Anil Kumar B. Polymers used in ophthalmic in situ gelling system. Int J Pharm Sci Rev Res 2013;20:176-83.

19. Gambhire S, Bhalerao K, Singh S. In situ hydrogel: different approaches to ocular drug delivery. Int J Pharm Pharm Sci 2013;5:27-36.

20. Champalal KD, Poddar SS. Current status of an ophthalmic insitu forming hydrogel. Int J Pharm Bio Sci 2012;3:372-88.

21. Kirti M, Shashikant D. In-situ gels-a novel approach for ocular drug delivery. World J Pharm Pharm Sci 2014;3:317-33.

22. Verma L, Sakir M, Singh N, Gilhotra RM, Mehan S. Development of phase change solutions for ophthalmic drug delivery based on ion activated and $\mathrm{pH}$-induced polymers. Int J Pharma Professional's Res 2010;1:127-34.

23. Singh J, Ankita K, Ghanshyam DG. In situ gelling system and recent trends in ocular drug delivery system. Indo Am J Pharm Res 2015;5:2306-15.

24. Gupta H, Aqil M, Khar RK, Ali A, Bhatnagar A, Mittal G, et al. Development and characterization of $99 \mathrm{mTc}$-timolol maleate for evaluating efficacy of in situ ocular drug delivery system. AAPS PharmSciTech 2009;10:540-6.

25. Makwana SB, Patel VA, Parmar SJ. Development and characterization of in-situ gel for an ophthalmic formulation containing ciprofloxacin hydrochloride. Results Pharma Sci 2016;6:1-6.

26. Preethi BG, Narendra E. Formulation and evaluation in-situ mucoadhesive ophtalmic hydrogel for sustained delivery of pefloxacin mesylate. Int J Pharm Pharm Sci 2015;7:345-50.

27. Vodithala S, Khatry S, Sadanandam NSM. Development and evaluation of thermoreversible ocular gels of ketorolac tromethamine. Int J Biopharm 2010;1:39-45.

28. Kavitha K, Rajas NJ. Sustained ophtalmic delivery of levofloxacin hemihydrate from an ion activated in situ gelling system. Int J PharmTech Res 2011;3:702-6.

29. Suresh, Abhishek. pH sensitive in situ ocular gel: a review. J J Pharm Sci Bioscientific Res 2016;6:684-94.

30. Mitali Bodhankar M, Bhushan S, Bhoyar, Vinit V Agnihotri. Environmentally responsive ophthalmic gel formulation: Based on combination with natural polycationic copolymer. Int J Pharm Res Dev 2012;3:212-22.

31. Swati G, Vyas SP. Carbopol/Chitosan-based pH-triggered in situ gelling system for ocular delivery of timolol maleate. Sci Pharm 2010;78:959-76. 